\section{P152 INTERFERON-I ACTIVITY IN EARLY AND ESTBLISHED RHEUMATOID ARTHRITIS (RA) WITH AND WITHOUT CARDIOVASCULAR ABNORMALITIES IN CARDIAC MAGNETIC RESONANCE (CMR)}

${ }^{1}$ AN Burska*, ${ }^{1} G$ Harrison, ${ }^{1} Y$ El-Sherbiny, ${ }^{1}$ L-A Bissell, ${ }^{1} A$ Giollo, ${ }^{1} E M$ Hensor, ${ }^{2} G$ Fent, ${ }^{2} \mathrm{~B}$ Erhayiem, ${ }^{2} \mathrm{~J}$ Greenwood, ${ }^{2} \mathrm{~S}$ Plein, ${ }^{1} \mathrm{~J}$ Andrews, ${ }^{1} \mathrm{E}$ Vital, ${ }^{1} \mathrm{MH}$ Buch. 'Leeds Institute of Rheumatic and Musculoskeletal Medicine; ' ${ }^{2}$ Leeds Institute of Cardiovascular and Metabolic Medicine, University of Leeds, Leeds, UK

\subsection{6/annrheumdis-2018-EWRR2019.135}

Career situation of first and presenting author Post-doctoral fellow.

Introduction RA is a heterogenous disease and there is a substantial evidence to indicate the contribution of type I interferon (IFN-I) in 20\%-40\% of RA patients with possibly more local IFN $\beta$ role; compared to systemic IFN- $\alpha$ action in SLE. Patients with RA have an increased risk of cardiovasular disease (CVD) equivalent to type 2 diabetes, predominantly driven by excess atherosclerosis (ATS). Pre-clinical and human data suggest IFN-I plays a key role in the development of ATS. IFN-I has been shown to underlie cardiovascular (CV) abnormalities in SLE.

Objectives IFN-I is important at the initiation of the pathological processes in early RA and increased IFN-I activity is associated with CVD in RA.

Methods The Vital group recently published a continuous 2score IFN system, ${ }^{1}$ IFN scores A and B (as opposed to an often used categorical classification of IFN high/low). We applied this scoring system in a cohort of early $($ ERA $n=75)$ and established RA (EstRA $n=101$ ) as well as in HC $n=71$. Next RA patients were stratified for CVD using multi-parametric cardiac MRI (CMR) evaluation that included aortic distensibility (vascular stiffness), LV mass/BSA (LV geometry) and Myocardial T1 (indicating myocardial fibrosis) as follows: (i) 'ERA-no CVD' $n=37$ (no abnormalities on CMR), (ii) 'ERA-sub CVD' $n=37$ (at least one of the three parameters abnormal), (iii) 'EstRA-no CVD', $\mathrm{n}=14$ (iv) 'EstRA-sub CVD', $\mathrm{n}=54$ (v) 'RA-CVD', $\mathrm{n}=32$ (defined as per 'Major Adverse Cardiovascular Event'(MACE)).

Results We confirmed a higher IFN score B than A in RA patients, similar to the observation made in the original paper that developed the scoring system. Significantly higher IFN score A and B in was observed in ERA than in EstRA and HCs. There was no association between IFN scores and markers of inflmmation. Increase in expression of IFN score B was observed across a CVD continuum (i.e. from RA-no CVD to RA-sub CVD to RA-CVD) in EstRA but not in ERA.

Conclusions IFN-I may play a particularly important pathological role at time of development of disease (ERA). CVD stratification suggests that genes included in IFN Scores A and B may be implicated in the progression along a CVD continuum; and appearing to associate with a pro-atherogenic role. This observation only in EstRA may reflect CVD burden over time. If confirmed, these data imply multiple organ specificities for the IFN scores. Further work is planned to interrogate IFN status in RA-CVD, towards improved risk stratification and tailored management of CVD co-morbidity.

\section{REFERENCE}

1. El-Sherbiny YM, et al. Scientific Reports 2018;8(1):5793.

Disclosure of Interest None declared

\section{P154 LOCAL LOW DOSE RADIATION INDUCES SYSTEMIC IMMUNE ALTERATIONS IN TWO EXPERIMENTAL MODELS OF INFLAMMATORY ARTHRITIS}

${ }^{1} \mathrm{~L}$ Deloch*, ${ }^{1} \mathrm{M}$ Rückert, ${ }^{2} \mathrm{M}$ Seeling, ${ }^{2} \mathrm{~F}$ Nimmerjahn, ${ }^{1} \mathrm{R}$ Fietkau, ${ }^{1} \mathrm{~B}$ Frey, ${ }^{1}$ US Gaipl. ${ }^{1}$ Radiation Oncology, Universitätsklinikum Erlangen; ${ }^{2}$ Institute of Genetics, Universität Erlangen-Nürnberg, Erlangen, Germany

10.1136/annrheumdis-2018-EWRR2019.136

Career situation of first and presenting author Post-doctoral fellow.

Introduction Rheumatoid arthritis (RA) is a chronic, progressive, inflammatory autoimmune disease that mainly affects the joints with its hallmarks being synovial inflammation followed by cartilage and bone destruction. There are a plethora of treatment options available, however, not all patients respond properly. In these patients it is crucial to slow down bone loss and inflammation in a timely manner to prevent further damage. Here, a therapy with low-dose ionizing radiation, the so called low-dose radiotherapy (LD$\mathrm{RT}$ ), could be an additional option. Detailed knowledge on the underlying mechanisms of reduced bone destruction and immune-mediated pain levels in patients following LD-RT is still scarce. We already showed that LD-RT locally slows down disease progression in human TNF $\alpha$ transgenic (hTNF $\alpha \mathrm{tg}$ ) animals by mainly having an impact on bone metabolism.

Objectives We now aim to take a closer look on systemic immune-mediated effects of LD-RT in both, hTNF $\alpha$ tg mice, as a cytokine mediated model, and in the KRN serum transfer model, as an example for arthritogenic antibody dependence.

Methods hTNF $\alpha$ tg or serum-injected C57Bl/6 mice were locally irradiated with a single dose per fraction of $0.5 \mathrm{~Gy}$. After an observation period of 7 days (serum-injected mice) or 30 days (hTNF $\alpha$-tg mice), blood, bone marrow as well as hind paws and synovial fluid was taken and analyzed using multicolor flow cytometry or histomorphometry.

Results Treatment of hind paws of serum-injected C57Bl/6 mice with $1 \times 0.5 \mathrm{~Gy}$ resulted in a systemic immune modulation. In the peripheral blood, an increase of eosinophils and a decrease of B cells and NK cells were observed. In the bone marrow, the prominent alteration was a shift from CD8 + to CD4 + T cells in both, the irradiated and non-irradiated leg. Furthermore, dendritic cells were decreased. In hTNF $\alpha$ tg animals, inflammation was modulated in a systemic manner after local LD-RT, while bone protection was a local effect.

Conclusions We conclude that LD-RT is not only a valuable tool for locally reducing inflammation and bone loss in the affected joints, but also has beneficial systemic immune-mediated effects. The observed immune modulations in the peripheral blood were similar to those observed for patients treated with LD-RT within the observational IMMO-LDRT01 study (NCT02653079). In the future, placebo-controlled studies with high patient numbers are desirable to clinically prove LD-RT as therapy for RA.

Acknowledgements Supported by the German Federal Ministry of Education and Research (GREWIS, 02NUK017G and GREWIS-alpha, 02NUK050E).

Disclosure of Interest None declared. 